\title{
Hepatolenticular Degeneration Combined with Primary Antiphospholipid Syndrome: A Case Report
}

\author{
Penka A. Atanassova ${ }^{a}$ Marija S. Panchovska $^{\mathrm{b}}$ Plamen Tzvetanov $^{\mathrm{c}}$ Nedka T. Chalakova $^{\mathrm{a}}$ \\ Radka I. Masaldzhieva ${ }^{d}$ Borislav D. Dimitrov $^{\mathrm{e}}$ \\ Departments of a Neurology and ${ }^{b}$ Internal Diseases and Rheumatology, Medical University, Plovdiv, \\ ${ }^{\mathrm{c}}$ Department of Neurology, Medical University, Pleven, ${ }^{\mathrm{d}}$ Department of Psychiatry and Medical Psychology,

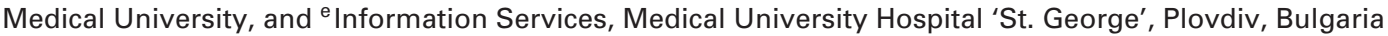

Dear Sir,

Hepatolenticular degeneration (HLD), also known as Wilson's disease, is an autosomal recessive defect in the metabolism of copper accompanied by a clinical constellation of symptoms of brain and liver damage $[1,2]$. Clinical progression can vary from favorable to untreatable depending on an early and accurate diagnosis [3]. Primary antiphospholipid syndrome (PAS) is an autoimmune disorder in which the antiphospholipid antibody production causes recurrent arterial and venous thrombosis, thrombocytopenia and symptoms of connective tissue disease $[4,5]$.

We report and discuss the unusual combination of HLD and PAS.

\section{Case Report}

First Presentation

A 34-year-old male patient, who had been healthy until the age of 31 , presented with increasing sensation of heaviness and rapid fatigue in the legs while walking continuously. His movements had become slower and he started stuttering. With these complaints, he was admitted in our Medical University Clinic of Neurology.

The neurological examination revealed dysarthria and a slightly increased plastic tone in the lower limbs. The laboratory tests revealed that the hemoglobin, erythrocytes, hematocrit, leukocytes, ESR and routine biochemical parameters were within the normal reference ranges. Thrombocytes were $77 \times 10^{9} / 1$; neither thrombocyte aggregation nor schistocytes were found on peripheral blood smear examination. The autoantibodies against ds-DNA, Sm, Ro/ SS-A, La/SS-B and RNP antigens (ELISA) and ANA titer (1:40 HEP-2) were negative. The antiphospholipid antibodies - IgG isotype (48 GPL) and IgM isotype (56 GPL) anticardiolipin antibodies - were elevated (ELISA) as well as those of $\beta_{2}$-glycoprotein (IgG 11.5, IgM 2.0). The lupus anticoagulant (LAC) test was positive while the Wassermann and antithrombotic antibody tests were negative. A repeat antiphospholipid antibody test after 3 months was again positive. The samples tested for the WaalerRose rheumatoid factor were negative. The neuroborreliosis examination was negative both in serum and cerebrospinal fluid. A bone marrow puncture was performed because of current thrombocytopenia: the cytologic examination evidenced bone marrow hyperplasia of hypercellular megakaryocytic type.

Liver and spleen ultrasonography demonstrated hepatosplenomegaly (liver at the right medioclavicular line $-152 \mathrm{~mm}$, homogeneous normoechogenic structure; cross-section size of spleen - $50 \mathrm{~mm}$ ). Brain magnetic resonance imaging (MRI) at
T2W/UTSE visualized microangiopathy (symmetrical hyperintense lesions in the zone of thalamus and lenticular nuclei bilaterally, presence of numerous foci in white matter with patterns of enlarged perivascular Virchow-Robin spaces). Thus we assumed that the discrete extrapyramidal signs were most probably associated with an antiphospholipid syndrome.

The patient's status was consistent with diagnostic criteria for PAS: thrombocytopenia and elevated levels of anticardiolipin antibodies (aCL) of $\mathrm{IgG}$ and $\mathrm{IgM}$ isotype which remained high in three different measurements and also tested positive for lupus anticoagulant [4]. The patient was referred to the Internal Diseases Clinic where he was given corticosteroid pulses and chronotherapy (Imuran, $50 \mathrm{mg} /$ day) for 2 years. After each corticosteroid administration, the patient reported 'some improvement' of his condition. Control laboratory parameters showed a decrease of his immunologic deficit, although mild thrombocytopenia and neurological symptomatology persisted.

\section{Re-Admission}

At the end of 3rd treatment year, the patient was readmitted to the Neurology Clinic with dominating extrapyramidal signs (bradykinesia, bradymimia, highly

\section{KARGER}

Fax +4161306 1234 E-Mail karger@karger.ch www.karger.com
(C) 2006 S. Karger AG, Basel 0014-3022/06/0551-0042\$23.50/0

Accessible online at: www.karger.com/ene
Penka A. Atanassova

Department of Neurology, Medical University

$15 \mathrm{~A}$ V. Aprilov

BG-4000 Plovdiv (Bulgaria)

Tel./Fax +359 32 649939, E-Mail pp_atanassova@yahoo.com 
unsteady walk, rigidly increased muscle tone in all limbs, postural instability, severe dysarthria - slurring and monotonous speech, dysphagia, hypersalivation, cerebellar ataxia, strong postural and intention tremor, greatly impaired fine movements of fingers), pyramidal signs (absent bilateral abdominal reflexes, positive Babinski sign bilaterally) and advancing cognitive deficit.

Control liver ultrasonography demonstrated hepatosplenomegaly with normoechogenic structure and no focal lesions. The newly performed tests showed: thrombocytes - $72.3 \times 10^{9} / 1$, serum copper $9.3 \mu \mathrm{g} / \mathrm{l}$, urine copper - $7.77 \mathrm{mmol} / \mathrm{l}$, ceruloplasmin $-0.112 \mathrm{~g} / \mathrm{l}$. The eye examination found bilateral greenish-yellow corneal depositions perilimbically, more pronounced in the left eye and characteristic of the Kayser-Fleischer ring. A new MRI examination showed no significant changes as compared with the previous one.

A psychological test found low average intelligence - IQ = 92 (Hamburg-WechslerIntelligenz Test). The patient's history of completed educational and professional levels suggested a previously higher level of intelligence.

The above findings based on extended clinical status with clear neurological symptoms as well as conclusive laboratory data positive for disturbed copper metabolism supported the diagnosis of HLD. The latter was 'masked' during the first hospitalization by the laboratory data for PAS and discrete neurological symptoms.

\section{Discussion}

After liver involvement, neurological manifestations are the second most frequent finding with initial clinical manifestation of HLD during the 3rd-4th decade of life, accounting for 40-60\% [1, 3] of newly diagnosed patients. Parkinson symptoms might result from a significant increase of copper concentration in the brain. However, nothing is known yet about the mechanisms of specific damage to the basal ganglia. The most frequently detected MRI abnormalities are changes in signal intensity of gray and white matter and atrophy of the caudate nucleus, brain stem and cerebellar hemispheres. Abnormalities in the striatum and pontocerebellar tract correlate with pseudoparkinsonian signs, whereas abnormalities in the dentatothalamic tract correlate with cerebellar signs [6]. For example, hyperintensities in the basal ganglia area, combined with atrophy of the cerebrum and cerebellum, were found in 28 patients [7]. The same regions were disturbed in our patient and thus correlated with extrapyramidal signs.

Basal ganglia are known to be affected in PAS. Extrapyramidal impairments (parkinsonism [8], dystonia [9, 10]) are rare. However, clinically established antiphospholipid syndrome with extrapyramidal symptoms has contributed to the initial diagnosis of PAS. In our patient, the diagnosis of HLD became plausible when a complete constellation of symptoms was formed, including the fully developed Parkinson syndrome, Kayser-Fleischer ring and low serum ceruloplasmin level. Various clinical cases had been reported where the psychiatric symptoms and cognitive deficit preceded the neurological manifestation of HLD, thus making it difficult to pose the correct diagnosis [14]. In our case we found only a slight cognitive decline at the end of the second year. We had a strong opinion in favor of PAS in such a young man who, in this view, had discrete neurological symptoms. This opinion indicated the prescription of immediate treatment for such an autoimmune disorder and, consecutively, the patient improved following the corticosteroid pulses.

A large number of hematological abnormalities are known to assist in the detection of HLD. Idiopathic thrombocytopenia [15], hemolytic anemia [16] and thrombotic thrombocytopenic purpura and hemolytic uremic syndrome $[17,18]$ are such rare cases as described earlier. To these diseases, which precede the full-blown picture of HLD, we can also add the present case of PAS. To the best of our knowledge, there is no reported case of such a combination, although there could be a possible pathophysiological link between these two diseases separately, as well as together, with the existing thrombocytopenia.

The case we have described is unique and very interesting because it is an example of a familial autosomal recessive disease (e.g., HLD) co-occurring with an autoimmune disorder (e.g., PAS) which does not belong to the group of collagen disorders. The neurological complications in thrombocytopenia can be a manifestation of both or either of the diseases. The fact that anticardiolipin antibody concentration was high during the follow-up and decreased steadily when the disease was treated adequately, did support the diagnosis of PAS.

\section{References}

1 Loudianos G, Gitlin JD: Wilson's disease. Semin Liver Dis 2000;20:353-364.

2 Adachi Y, et al: Presence of ATP-dependent copper transport in the hepatocyte canalicular membrane of the Long-Evans cinnamon rat, an animal model of Wilson disease. J Hepatol 1997;26:216-217.

3 El-Youssef M: Wilson disease. Mayo Clin Proc 2003;78:1126-1136.

4 Hughes GRV: The antiphospholipid syndrome. Lancet 1993;342:341-344.

5 Galve E, et al: Valvular heart disease in the primary antiphospholipid syndrome. Ann Intern Med 1992;166:293-298.

6 Van Wassenaer-van Hall HN, et al: Wilson disease: findings at MR imaging and $\mathrm{CT}$ of the brain with clinical correlation. Radiology 1996; 198:531-536.

7 Hermann W, et al: Cerebral MRI and evoked potentials in Wilson disease. Comparison of findings in patients with neurological followup. Nervenarzt 2002;73:349-354.

8 Milanov I, et al: Antiphospholipid syndrome and parkinsonism. Clin Exp Rheumatol 1998; 16:623-624.

9 Svetel M, et al: Dystonia in Wilson's disease. Mov Disord 2001;16:719-723.

10 Milanov I, Bogdanova D: Antiphospholipid syndrome and dystonia-parkinsonism. A case report. Parkinsonism Related Disord 2001;7: 139-141.

11 Demirkiran M, et al: Neurologic presentation of Wilson disease without Kayser-Fleischer rings. Neurology 1996;46:1040-1043.

12 Steindl P, et al: Wilson's disease in patients presenting with liver disease: a diagnostic challenge. Gastroenterology 1997;113:212-218.

13 Cauza E, Maier-Dobersberger Th, Ferenci P: Plasma ceruloplasmin as screening test for Wilson's disease. J Hepatol 1997;27:358-362.

14 Starosta-Rubinstein S, et al: Clinical assessment of 31 patients with Wilson's disease. Arch Neurol 1987;44:365-370.

15 Donfrid M, et al: Idiopathic thrombocytopenia associated with Wilson's disease. Hepatogastroenterology 1998;45:1774-1776.

16 Prella M, et al: Haemolytic onset of Wilson disease in a patient with homozygous truncation of ATP7B at Arg 1319. Br J Haematol 2001; 114:230-232.

17 Szotkowski T, et al: Thrombotic thrombocytopenic purpura - hemolytic uremic syndrome (TTP-HUS) leading to the diagnosis of Wilson's disease. Vnitr Lek 2002;48:353-356.

18 Ruggenenti P, Noris M, Remuzzi G: Thrombotic microangiopathy, hemolytic uremic syndrome, and thrombotic thrombocytopenic purpura. Kidney Int 2001;60:831-846. 